Tissue transglutaminase (TG2) activity regulates osteoblast differentiation and mineralization in the SAOS-2 cell line

Xiaoxue Yin, Zhongqiang Chen, Zhongjun Liu and Chunli Song

The Brazilian Journal of Medical and Biological Research is partially financed by

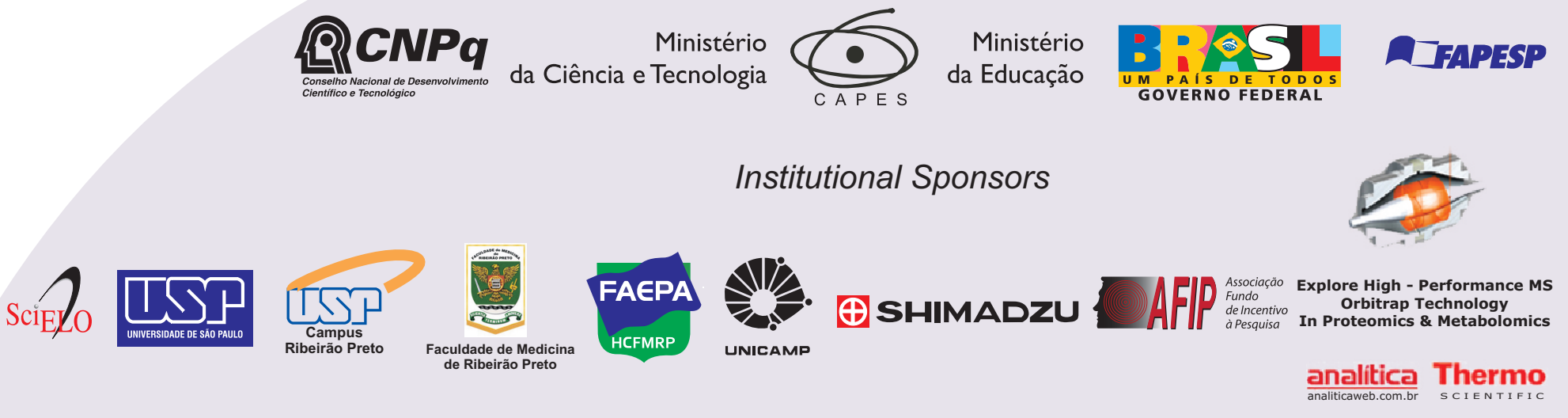




\title{
Tissue transglutaminase (TG2) activity regulates osteoblast differentiation and mineralization in the SAOS-2 cell line
}

\author{
Xiaoxue Yin, Zhongqiang Chen, Zhongjun Liu and Chunli Song \\ Department of Orthopaedics, Peking University Third Hospital, Beijing, China
}

\begin{abstract}
Tissue transglutaminase (type II, TG2) has long been postulated to directly promote skeletal matrix calcification and play an important role in ossification. However, limited information is available on the expression, function and modulating mechanism of TG2 during osteoblast differentiation and mineralization. To address these issues, we cultured the well-established human osteosarcoma cell line SAOS-2 with osteo-inductive conditioned medium and set up three time points (culture days 4, 7, and 14 ) to represent different stages of SAOS-2 differentiation. Osteoblast markers, mineralization, as well as TG2 expression and activity, were then assayed in each stage. Furthermore, we inhibited TG activity with cystamine and then checked SAOS-2 differentiation and mineralization in each stage. The results showed that during the progression of osteoblast differentiation SAOS-2 cells presented significantly high levels of osteocalcin (OC) mRNA, bone morphogenetic protein-2 (BMP-2) and collagen I, significantly high alkaline phosphatase (ALP) activity, and the increased formation of calcified matrix. With the same tendency, TG2 expression and activity were up-regulated. Furthermore, inhibition of TG activity resulted in a significant decrease of OC, collagen I, and BMP-2 mRNA and of ALP activity and mineralization. This study demonstrated that TG2 is involved in osteoblast differentiation and may play a role in the initiation and regulation of the mineralization processes. Moreover, the modulating effects of TG2 on osteoblasts may be related to BMP-2.
\end{abstract}

Key words: Transglutaminase; Osteoblast; Differentiation; Mineralization; Bone morphogenetic protein-2 (BMP-2)

\section{Introduction}

Transglutaminase enzymes (TGs; EC 2.3.2.13) are a group of enzymes whose main function is to stabilize and assemble their substrate proteins into large polymers by creating covalent $\gamma$-(glutamyl)- $\varepsilon$-lysyl bonds (i.e., isopeptide crosslinks) between glutamine and lysine residues in a calcium ion-dependent reaction (1). To date, the transglutaminase family consists of 9 different TG genes (2). Tissue transglutaminase (TG2, type II) is one of the best-characterized, skeletal tissue-related TGs $(3,4)$ and may be involved in the initiation of mineralization and play an important role in ossification $(5,6)$.

The biological function of TG2 has yet to be determined. However, there is now increasing evidence suggesting that TG2 can act at the cell surface facilitating cell adhesion, cell spreading and the modification of the extracellular matrix $(E C M)(7,8)$. Moreover, TG2 is a highly selective enzyme, with only a few native proteins identified as its substrates (9). In mineralized tissues, collagen, fibronectin, osteopontin, and bone sialoprotein are all TG2 substrates, which assemble into polymeric forms to participate in matrix stabilization, chondrocyte and osteoblast differentiation and matrix mineralization in the presence of TG2 (3).

TG2 is expressed in cartilage, bone, and teeth, the most extensively studied being cartilage $(3,5,10,11)$. It has been demonstrated that TG2 expression correlates with chondrocyte differentiation and matrix mineralization. Moreover, chondrocyte cultures from TG2-knockout mice show lack of induction of matrix calcification (12), while addition of exogenous TG2 increases chondrocyte hypertrophy and mineralization $(10,13)$.

TG2 enzyme activity is also found in extracts of intramembranous bone, and has been identified in osteoblast-like bone cells in vitro, with results suggesting an involvement in bone cell adhesion and ECM calcification (3,14,15). Al-Jallad et al. (16) demonstrated that the TG genes expressed and active in MC3T3-E1 pre-osteoblast cultures are TG2 and

Correspondence: Zhongqiang Chen, Department of Orthopaedics, Peking University Third Hospital, \#49 Hua-Yuan North Road, Haidian District, Beijing 100191, China. Fax:+8610-8226-5557. E-mail: luckyemail2008@sina.com or zhongqiangchen@tom.com

Received November 25, 2011. Accepted April 9, 2012. Available online April 27, 2012. Published August 3, 2012. 
FXIIIA, and Wosniak et al. (15) showed that mechanical strain increased osteopath mineralization by the action of TG2. In addition, the TG-catalyzed N $\varepsilon$ ( $\gamma$-glutamyl) lysine crosslinks are abundant in the bone matrix and also seem to correlate with ectopic mineralization in pathological processes such as atherosclerosis (17) and osteoarthritis (18). Our previous study also showed that TG2 expression and enzyme activity are up-regulated in the ossification cells of the ligamentum flavum (OLF) and TG2 may be involved in the pathologic process of OLF (19). Collectively, these findings indicate that TG2 activity is critical to osteoblast differentiation and matrix maturation.

Although TG2 has been noted in osteoblasts, limited information is available on the expression, function and modulating mechanism of TG2 during osteoblast differentiation and mineralization. We intended to address these issues in vitro using the well-established, matrix-producing and mineralizing human osteosarcoma cell line SAOS-2 (20). In this study, we set up three times (days 4, 7, and 14) to represent different stages of SAOS-2 differentiation and mineralization, and assayed TG2 expression and activity in each stage. Furthermore, we inhibited TG activity with cystamine and then checked SAOS-2 differentiation and subsequent mineralization in each stage. The results provided insight into the contribution of TG2 to the cascade of events leading to bone differentiation and matrix maturation.

\section{Material and Methods}

\section{Cell culture}

SAOS-2 cells were a generous gift from Dr. David R. Eyre (University of Washington Medical Center, Seattle, WA, USA). Cells were routinely grown in Dulbecco's modified Eagle's medium (DMEM; Gibco, USA), supplemented with $10 \%$ fetal bovine serum (Gibco), and incubated in a humidified atmosphere of $95 \%$ air and $5 \% \mathrm{CO}_{2}$ at $37^{\circ} \mathrm{C}$. The culture medium was changed every second day. Differentiation and mineralization were induced by the osteoinductive conditioned medium, which was composed of $10 \%$ FBS DMEM supplemented with $50 \mu \mathrm{g} / \mathrm{mL}$ L-ascorbic acid (AA; Sigma, USA) and $10 \mathrm{mM} \beta$-glycerophosphate ( $\beta$-GP,
Sigma). Cells treated with $10 \%$ FBS DMEM normal medium were used as controls for all experiments.

\section{Osteoblast differentiation and mineralization assays}

SAOS-2 cells were cultured in osteo-inductive conditioned medium for 4,7 , and 14 days and were then harvested individually. Subsequent assays of osteoblast differentiation and mineralization were carried out as described below.

\section{mRNA expression of osteoblast markers}

Total RNA of cells was extracted with Trizol reagent. cDNA synthesis was carried out with $5 \mu \mathrm{g}$ total RNA, $1 \mu \mathrm{L}$ random primer, $2 \mu \mathrm{L}$ dNTPs and $200 \mathrm{U}$ M-MLV reverse transcriptase (Promega, USA) at $37^{\circ} \mathrm{C}$ for $1 \mathrm{~h}$. The osteocalcin (OC), bone morphogenetic protein-2 (BMP-2), collagen I, and TG2 genes in mRNA were detected by semiquantitative RT-PCR, with the amplification of $\beta$-actin as an internal control. The specific primers (Invitrogen, USA) were designed by Primer Premier and described in Table 1.

Amplification was performed using the Platinum PCR SuperMix (Invitrogen). PCR was carried out for 33 cycles, each at $95^{\circ} \mathrm{C}$ for $30 \mathrm{~s}$, at $58^{\circ} \mathrm{C}$ for $30 \mathrm{~s}$, at $72^{\circ} \mathrm{C}$ for $1 \mathrm{~min}$, with a final extension at $74^{\circ} \mathrm{C}$ for $7 \mathrm{~min}$. The PCR products were analyzed by $3 \%$ agarose gel electrophoresis and visualized with ethidium bromide staining, and the densitometry values of the bands were quantified and analyzed using the Image J software.

\section{Alkaline phosphatase activity assay}

Alkaline phosphatase (ALP) activity was assayed using the LabAssay ${ }^{\mathrm{TM}}$ ALP kit (Wako, Japan). SAOS-2 cells were seeded onto 6 -well plates at a density of $2 \times 10^{5}$ cells per well and treated for the designated time. Cells were then obtained and completely lysed by sonication for 10 min with a sonifer cell disruptor (Cosmo Bio, Japan). The sonicates were centrifuged for $10 \mathrm{~min}$ at 20,142 $\mathrm{g}$, and the supernatants were used as samples for the ALP activity assay. The protein concentrations were determined with the bicinchoninic (BCA) protein assay reagent. The relative activity of the sample is reported as the ratio of activity and the corresponding protein concentration $(\mathrm{U} / \mathrm{mg})$.

Table 1. Primers for RT-PCR used to detect osteoblast markers and TG2 in SAOS-2.

\begin{tabular}{llcll}
\hline Target & Accession No. & Product size & \multicolumn{1}{c}{ Upstream primer } & \multicolumn{1}{c}{ Downstream primer } \\
\hline TG2 & NM_004613 & 167 & GGGGTGAGAGAGGAAAGACC & TGCAGTCTAGGGAGCTGGAT \\
BMP-2 & M_001200 & 197 & TCAAGCCAAACACAACACGC & ACGTCTGAACAATGGCATGA \\
Osteocalcin & NM_199173 & 230 & GGCAGCGAGGTAGTGAAGAG & CTGGAGAGGAGCAGAACTGG \\
Collagen I & NM_000088 & 381 & GGAGGGAATCACTGGTGCTA & AGGGGGAAAACTGCTTTGT \\
ß-actin & NM_001101 & 275 & CAGGAGATGGCCACTGCCGCA & TCCTTCTGCATCCTGTCAGCA \\
\hline
\end{tabular}

TG2 = transglutaminase type II; BMP-2 = bone morphogenetic protein-2 


\section{Mineralization assay}

Calcified nodules on the cells were determined by Alizarin red staining. SAOS-2 cells were seeded into $3.5-\mathrm{cm}$ dishes at a density of $2 \times 10^{5}$ cells per dish and treated for the designed time. After incubation, the cells were rinsed with PBS (without $\mathrm{Mg}^{2+}$ and $\mathrm{Ca}^{2+}$ ) and fixed in ice-cold 95\% ethanol for $30 \mathrm{~min}$ at $-20^{\circ} \mathrm{C}$. Subsequently, the cells and the matrix were stained with $40 \mathrm{mM}$ Alizarin red-S, $\mathrm{pH} 4.2$, for 1 $\mathrm{h}$ at room temperature. The stained nodules that appeared bright red in color represented physiological mineralization but not dystrophic mineralization, and they were identified by light microscopy. To compare the stained region of mineralization, the whole dish was photographed.

\section{TG2 enzyme assay}

Protein expression of TG 2 by Western blot. SAOS- 2 cells were cultured on 100-mm culture dishes for the designed time. Total cellular protein was isolated from cultured cells using radio-immunoprecipitation assay extraction buffer. Protein concentration was determined with the BCA protein assay reagent.

A total of $25 \mu \mathrm{g}$ protein was loaded per well, separated on $10 \%$ SDS-PAGE gels and subsequently transferred to polyvinylidene fluoride membranes. Membranes were blocked overnight at $4{ }^{\circ} \mathrm{C}$ with $3 \%$ bovine serum albumin/ Tris-buffered saline and Tween 20 (BSA/TBST) and then incubated with primary antibodies against TG2 (rabbit polyclonal antibody, sc-20621, Santa Cruz Biotechnology Inc., USA), followed by the secondary antibody (IRDye 800cw conjugated Goat (polyclonal) anti-rabbit IgG, 926-32211, LI-COR Biosciences, USA). Bands were visualized using an infrared fluorescent scan imaging system (Odyssey, USA). GAPDH was used as the internal control. The densitometry values of the fluorescent bands were quantified and analyzed using the Image $\mathrm{J}$ software.

\section{TG2 enzyme activity assay}

SAOS-2 cells were cultured on 100-mm culture dishes for the designated time. TGase activity was measured by a previously described method (19). Specifically, we coated 96-well ImmunoModule plates with $200 \mu \mathrm{L} 20$ mg/ $\mathrm{mL} \mathrm{N}, \mathrm{N}$-dimethylcasein for $1 \mathrm{~h}$ at $23^{\circ} \mathrm{C}$. The N,N-dimethylcasein was removed and nonspecific protein binding was blocked by adding 3\% BSA in $100 \mathrm{mM}$ Tris, $\mathrm{pH} 8.5$, $150 \mathrm{mM} \mathrm{NaCl}, 0.05 \%$ Tween-20 (TBST) to each well for an additional $1 \mathrm{~h}$ at $23^{\circ} \mathrm{C}$. Subsequently, aliquots of $25 \mu \mathrm{g}$ total cellular protein that had been lysed and sonicated (in $5 \mathrm{mM}$ Tris- $\mathrm{HCl}, 0.25 \mathrm{M}$ sucrose, $0.2 \mathrm{mM} \mathrm{MgSO}_{4}, 2 \mathrm{mM}$ dithiothreitol, $0.4 \mathrm{mM}$ phenylmethyl sulfonyl fluoride, $0.4 \%$ Triton $\mathrm{X}-100, \mathrm{pH} 7.5)$ were added to the plate in triplicate. Fifty microliters of solution A (100 mM Tris, $\mathrm{pH} 8.5$, and $20 \mathrm{mM} \mathrm{CaCl}_{2}$ ) was added to all samples, followed by the addition of $50 \mu \mathrm{L}$ of solution $\mathrm{B}(100 \mathrm{mM}$ Tris, $\mathrm{pH} 8.5,40$ $\mathrm{mM}$ dithiothreitol, and freshly added $2 \mathrm{mM} 5$-(biotinamido) pentylamine). The plates were incubated for $1 \mathrm{~h}$ at $37^{\circ} \mathrm{C}$.
The wells were washed once with TBST containing $1 \mathrm{mM}$ ethylenediaminetetraacetic acid and then three times with TBST. One hundred microliters of a 1:5000 dilution of streptavidin-peroxidase in 3\% BSA/TBST was added to each well for $1 \mathrm{~h}$ at $23^{\circ} \mathrm{C}$. The wells were washed twice with TBST, and $100 \mu \mathrm{L}$ TMB working solution was added to each well. Absorbance was measured at $450 \mathrm{~nm}$ for 15 min after adding $0.5 \mathrm{M} \mathrm{H}_{2} \mathrm{SO}_{4}$ to stop the reaction. Purified guinea pig liver TGase (Sigma) was used to prepare a standard curve. TG enzyme activity was designated as the amount of 5-(biotinamido)pentylamine incorporated into casein.

\section{SAOS-2 differentiation and mineralization assay when TG activity was inhibited by cystamine}

SAOS-2 cells were seeded on 100-mm dishes (for RTPCR) or 48-well plates (for ALP assay) or 3.5-cm dishes (for Alizarin red staining) with osteo-inductive conditioned medium and were allowed to attach overnight. The cells were then treated with $0.5 \mathrm{mM}$ cystamine (Sigma-Aldrich, USA) for the indicated days, i.e., 0-14 or 4-14. Cells cultured with conditioned medium but without cystamine were used as controls and cells cultured with normal medium as the blank control. Collagen I, OC, and BMP-2 mRNA were assayed by semiquantitative RT-PCR, ALP activity with the LabAssay ${ }^{\mathrm{TM}}$ ALP kit and mineralization by Alizarin red staining.

\section{Statistical analysis}

Data were analyzed for statistical significance by oneway ANOVA using the Dunnett test and the SPSS software. $P<0.05$ was considered to be statistically significant.

\section{Results}

\section{Expression of TG2 by SAOS-2 cells}

To screen TG2 expression and activity during the osteoblast program, we set up three time points (days 4, 7, and 14) to represent different stages of SAOS-2 cultures (with $A A+\beta-G P$ ), and cultures without $A A$ and $\beta-G P$ were used as controls. We observed that day 4 might be the early stage of osteoblast differentiation when cellular deposition of collagen had just begun to form a matrix and the expressions of OC and collagen I mRNA, as well as ALP activity, were almost as low as those observed for the controls. Moreover, there was nearly no positive staining for calcified matrix with Alizarin red staining at this stage, although BMP-2 expression appeared to increase significantly compared to control. Day 7 was a differentiation stage with statistically significant high expression of BMP-2 and collagen I, as well as dramatically high ALP activity and the increased formation of calcified matrix. Day 14 represented the fully differentiated and mineralized stage with abundant calcified matrix and significantly increased OC, BMP-2 and collagen I mRNA and ALP activity (Figure 1A-D). 
Semiquantitative RT-PCR and Western blot were performed to examine TG2 mRNA and its protein expression at the three differentiation stages. TG2 mRNA expression appeared to be up-regulated with the progression of osteoblast differentiation. Compared to the controls and to the early stage at day 4, TG2 mRNA significantly increased at days 7 and 14 (Figure 2A). Furthermore, the results of Western blot showed that TG2 mRNA was transcribed into protein and the protein levels were significantly high at days 7 and 14, with the same tendency observed for TG2 mRNA level (Figure 2B).

TG2 activity in SAOS-2 was dramatically increased when cultured with osteo-inductive conditioned medium, even at the early stage of day 4 . Compared to control cultures, TG2 activity was enhanced by about 3- to 4-fold when treated with conditioned medium, which was inde- pendent of culture time (Figure 2C).

\section{Inhibition of TG activity blocks osteoblast differentiation and mineralization}

To gain insight into the function of TG2 in the osteoblast program, we inhibited TG activity during cell differentiation and mineralization with the well-known TG inhibitor cystamine (CYS) (21). CYS treatment $(0.5 \mathrm{mM})$ does not disturb cell growth (16). However, our data showed that the treatment resulted in a significant decrease of OC, collagen I and BMP-2 mRNA, an obvious reduction of ALP activity and disturbance of mineralization, regardless of the inhibition of CYS between days 4 and 14, or between days 0 and 14. But the inhibition for the full 14-day period was more effective in blocking mineral deposition as visualized directly by the absence of Alizarin red staining (Figure $3 A-C$ ).
A
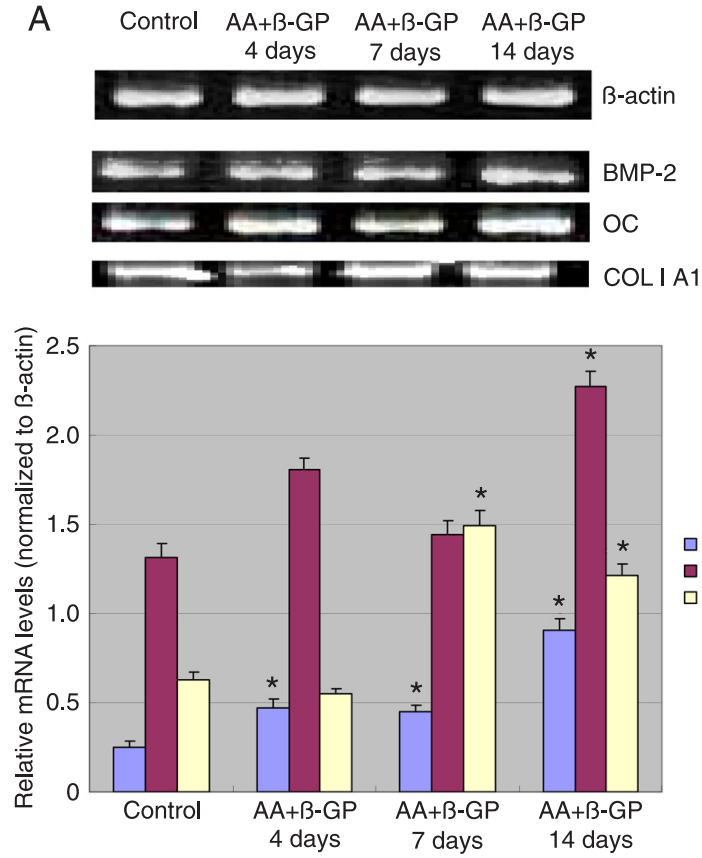

B

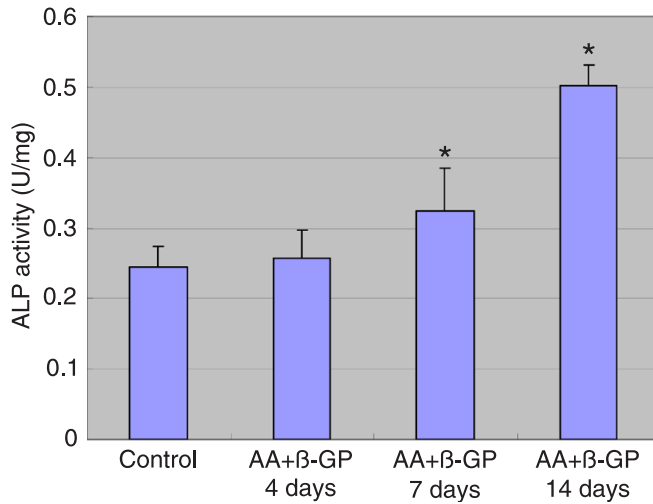

C
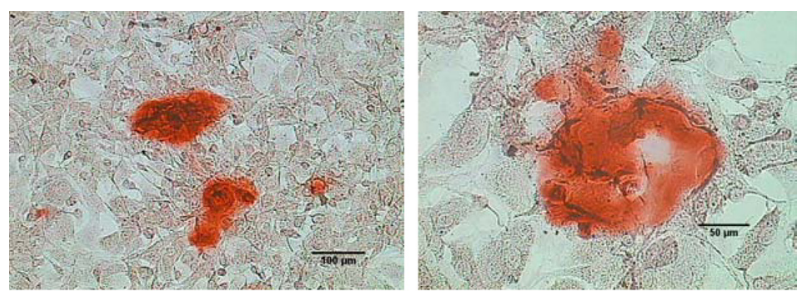

$\mathrm{D}$

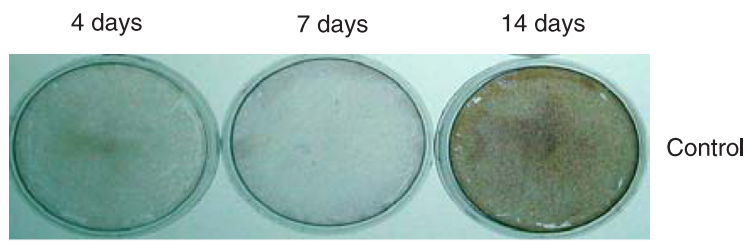

$\square O C$

$\square \mathrm{COL}$ IA1

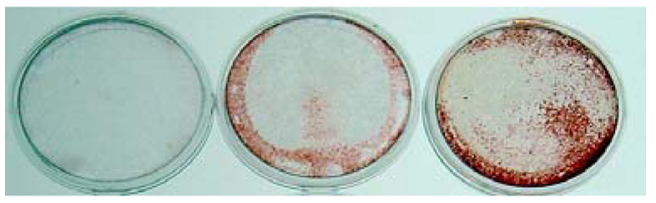

$A A+B-G P$

Figure 1. Differentiation and mineralization of SAOS-2 cells cultured in osteo-inductive conditioned medium supplemented with AA and $\beta$-GP for 14 days. Control cultures were treated with normal medium only, without the above additives. $A, \mathrm{mRNA}$ expression of the osteoblast markers $\mathrm{COL}$ $\mathrm{I}, \mathrm{OC}$, and BMP-2 in the cultures over a period of 14 days as analyzed by semiquantitative RT-PCR. The densitometry quantification of the electrophoretic profiles was normalized to the corresponding $\beta$-actin. Data were combined from 3 duplicate experiments and reported as means \pm SEM. ${ }^{*} \mathrm{P} \leq 0.05$ vs control (Dunnett test). B, ALP activity during differentiation measured colorimetrically using $\mathrm{p}$-nitrophenylphosphate as a substrate. Data were combined from 6 duplicate experiments and reported as means \pm SEM. * $\mathrm{P} \leq 0.05$ vs control (Dunnett test). $\mathrm{AA}=\mathrm{L}$-ascorbic acid; $\beta$-GP $=$ $\beta$-glycerophosphate; COL I = collagen I; OC = osteocalcin; BMP-2 = bone morphogenetic protein-2. $C$, Light microscopy of the stained calcified nodules assayed by Alizarin red staining. $D$, Mineralization (stained region) of the cultures as assessed by Alizarin red staining. 

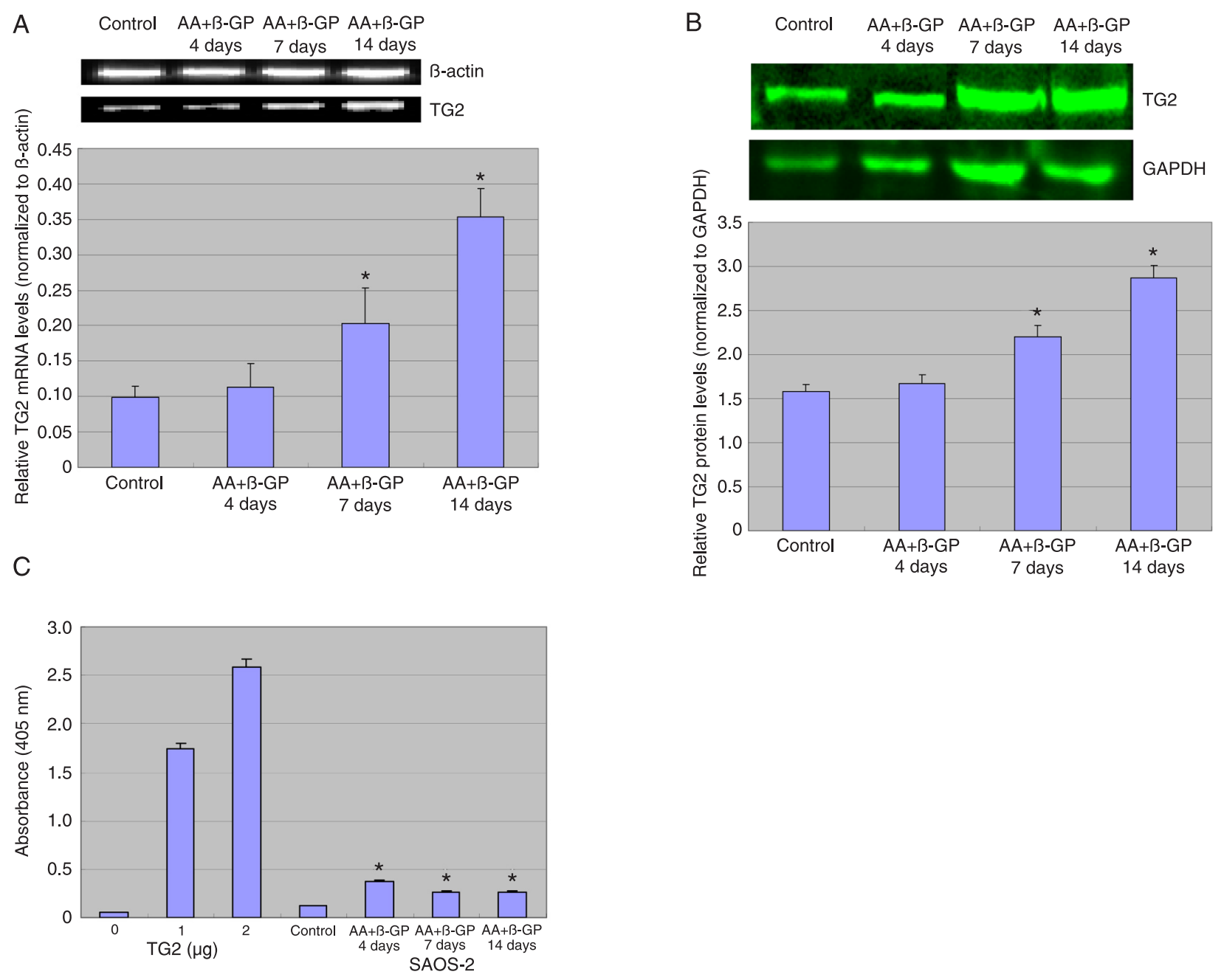

Figure 2. Transglutaminase type II (TG2) mRNA, protein expression and enzyme activity in SAOS-2 cells during osteoblast differentiation and mineralization. A, Semiquantitative RT-PCR analysis of TG2 genes from SAOS-2 cells cultured in osteo-inductive conditioned medium at days $4,7,14$, which represent different stages of osteoblast differentiation and mineralization, or in normal medium for 14 days (control). The densitometry quantification of the electrophoretic profiles was normalized to the corresponding $\beta$-actin. Data were combined from 3 duplicate experiments and are reported as means $\pm \mathrm{SEM}$. ${ }^{*} \mathrm{P} \leq 0.05$ vs control (Dunnett test). $B$, Western blot analysis of TG2 protein expression in SAOS-2 cells at different stages of differentiation. Control cells were grown without L-ascorbic acid (AA) and $\beta$-glycerophosphate ( $\beta$-GP) for 14 days. The densitometry quantification of the fluorescent bands was normalized to the corresponding GAPDH. Data were combined from 3 duplicate experiments and are reported as means $\pm S E M$. ${ }^{+} P \leq 0.05$ vs control (Dunnett test). C, TG2 activity of SAOS-2 cells at different stages of differentiation. Control cells were grown without AA and $\beta-G P$ for 14 days. The results were compared and contrasted to the signal obtained with 0 to $2 \mu \mathrm{g}$ purified guinea pig TG2. Data were combined from 6 duplicate experiments and are reported as means \pm SEM. ${ }^{*} \mathrm{P} \leq 0.05 \mathrm{vs}$ control (Dunnett test).

\section{Discussion}

SAOS-2 is an established human osteosarcoma cell line, which possesses a typical osteoblastic phenotype, including elevated ALP, parathyroid hormone-stimulatable adenylate cyclase, synthesis and secretion of type I collagen, OC, and osteopontin, and production of mineralized matrix (20). SAOS-2 represents a useful experimental model for studying osteoblastic properties and osteoblast-produced molecules and is supposed to provide significantly more information than a mouse osteoblast cell line. When the cell line is cultured with osteo-inductive conditioned medium supplemented with $50 \mu \mathrm{g} / \mathrm{mL}$ AA and $10 \mathrm{mM} \beta-\mathrm{GP}$, osteoblasts will be induced to differentiate and to form calcified matrix $(16,22)$. In our study, we consistently observed that during 14 days of culture in conditioned medium, SAOS-2 cells exhibited the typical early differentiation stage (day 4), differentiation stage (day 7) and fully differentiated and mineralized stage (day 14) of osteoblasts. More interestingly, although Al-Jallad et al. (16) reported that TG2 
A
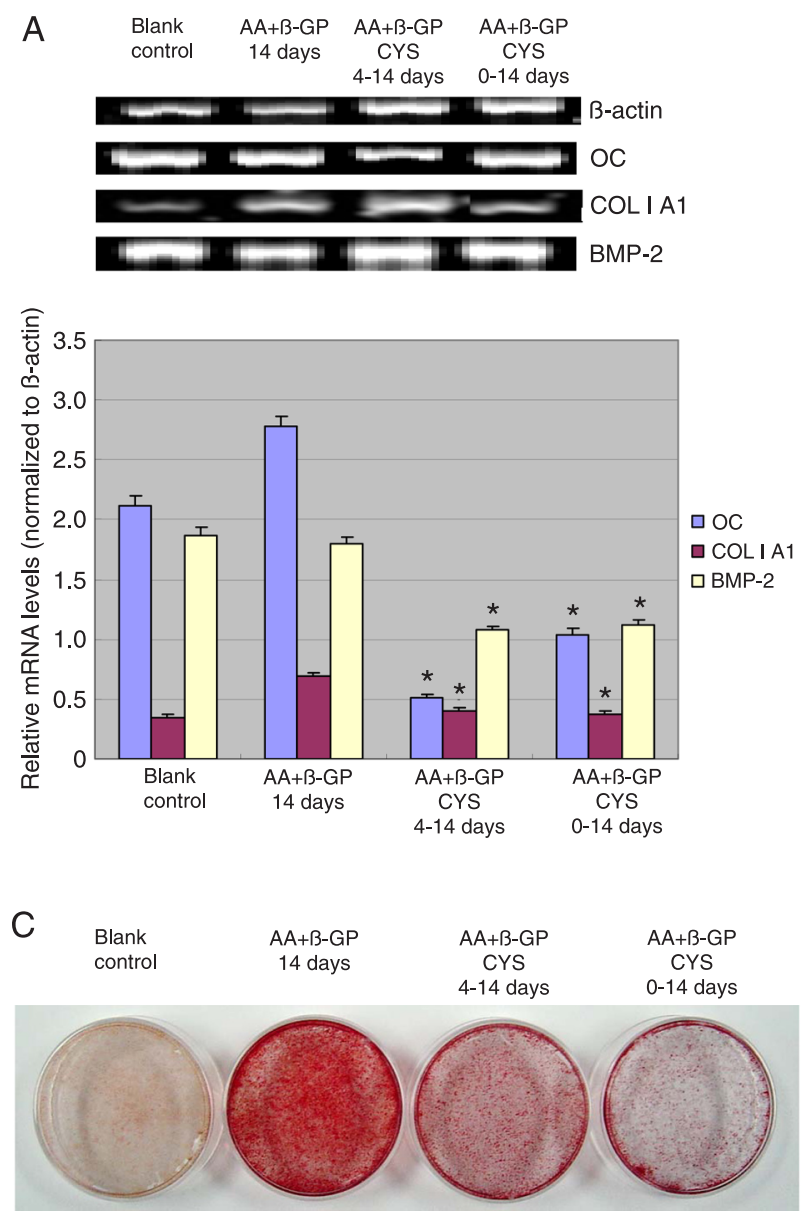

$\mathrm{B}$

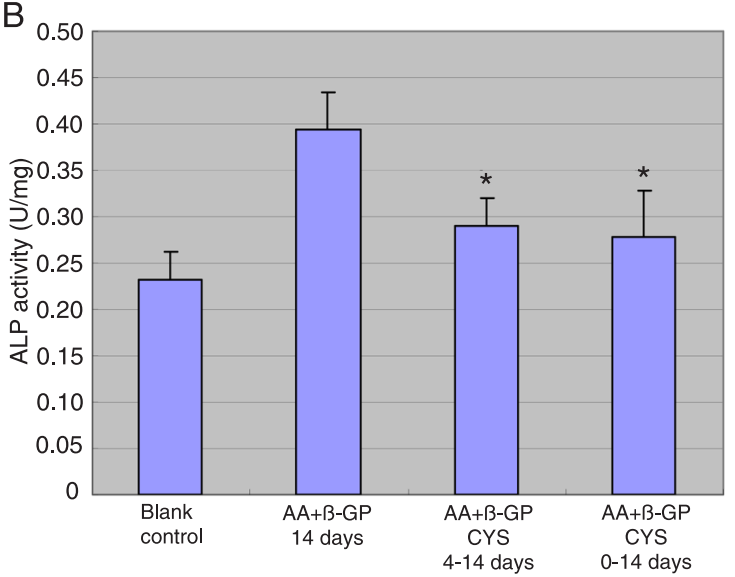

Figure 3. Inhibition of TG2 activity by cystamine (CYS) blocks the differentiation and mineralization of SAOS-2 cells. Cells were cultured in conditioned medium and treated with $0.5 \mathrm{mM}$ CYS for the indicated days: 0-14 and 4-14. Control cells were cultured with conditioned medium without CYS and blank control cells were cultured with normal medium without CYS for 14 days. $A$, mRNA expression of the osteoblast markers COL I, OC, and BMP-2 in the cultures was analyzed by semiquantitative RT-PCR. The densitometry quantification of the electrophoretic profiles was normalized to the corresponding $\beta$-actin. Data were combined from 3 duplicate experiments and are reported as means \pm SEM. COL I = collagen I; OC = osteocalcin; BMP $-2=$ bone morphogenetic protein-2. ${ }^{*} \mathrm{P} \leq 0.05$ vs control (Dunnett test). $B$, Alkaline phosphatase (ALP) activity was measured colorimetrically using p-nitrophenylphosphate as a substrate. Data were combined from 6 duplicate experiments and reported as means \pm SEM. ${ }^{*} \mathrm{P} \leq$ 0.05 vs control (Dunnett test). C, Mineralization (stained region) of the cultures as assessed by Alizarin red staining.

mRNA and protein levels remain constant throughout the differentiation program in MC3T3-E1 cells, we found that in this human osteoblast cell line, the expression of TG2 protein and mRNA, which was consistent with the expression of osteoblast markers, was dramatically elevated with the progression of osteoblast differentiation, especially at the fully differentiated and mineralized stage. Furthermore, TG2 activity responded (increased) to the osteo-inductive conditioned medium. Even in the early differentiation phase (day 4), TG2 activity was increased by about 3-fold in cells cultured with conditioned medium, as compared to cells cultured with normal medium. Furthermore, we demonstrated that when TG (including TG2) activity was inhibited by CYS, the expression of osteoblast markers, ALP activity and mineralization of SAOS-2 was reduced synchronously and significantly. Compared to inhibition between day 4 and day 14 , the full 14-day inhibition was more effective, resulting in an almost complete block of mineralization. This suggested that TG2 activity at the early phase (0-4 days) is very important for mineralization. Collectively, our results directly linked the progression of osteoblast differentiation and mineralization to the up-regulated TG2, demonstrating that TG2 is involved in osteoblast differentiation and may play a role in the initiation and regulation of the mineralization processes.

Among 9 family members of TGs, TG2 and FXIIIA have long been linked to the formation of skeletal elements, and growing evidence indicates that these two enzymes have similar and/or overlapping, but not necessary identical, functions in connective tissue cells (23). However, Al-Jallad 
et al. (16) reported that FXIIIA could play a major role in MC3T3-E1 cell differentiation and collagen I matrix formation, and TG2 crosslinking activity did not contribute to osteoblast differentiation $(16,24)$. However, in the current study, although we did not assay the expression of FXIIIA, we did find that TG2 levels continued to increase steadily during osteoblast differentiation. The difference might be attributed to the diversity of two different cell lines. Alternatively, human osteoblasts may have a different mechanism than mouse osteoblasts to activate TGase activity. Additional effort is now being directed towards understanding the difference of TG2 effect by using different cell lines from different species and also primary cells.

It has been demonstrated that TG2 has some main physiological functions independent of its crosslink activity, such as signal transduction, cell adhesion and interaction with transforming growth factor-beta (TGF- $\beta$ ) $(1,25)$. Our results showed that during the progression of SAOS-2 differentiation, BMP-2 mRNA increased significantly and TG2 expression and activity were up-regulated at the same time. However, when TG2 was inhibited, BMP-2 expression decreased dramatically. As we all know, BMP-2 belongs to the

\section{References}

1. Lorand L, Graham RM. Transglutaminases: crosslinking enzymes with pleiotropic functions. Nat Rev Mol Cell Biol 2003; 4: 140-156.

2. Grenard P, Bates MK, Aeschlimann D. Evolution of transglutaminase genes: identification of a transglutaminase gene cluster on human chromosome 15q15. Structure of the gene encoding transglutaminase $X$ and a novel gene family member, transglutaminase Z. J Biol Chem 2001; 276: 33066-33078.

3. Kaartinen MT, El-Maadawy S, Rasanen NH, McKee MD. Tissue transglutaminase and its substrates in bone. J Bone Miner Res 2002; 17: 2161-2173.

4. Nurminskaya M, Kaartinen MT. Transglutaminases in mineralized tissues. Front Biosci 2006; 11: 1591-1606.

5. Rosenthal AK, Masuda I, Gohr CM, Derfus BA, Le M. The transglutaminase, Factor XIIIA, is present in articular chondrocytes. Osteoarthritis Cartilage 2001; 9: 578-581.

6. Thomazy VA, Davies PJ. Expression of tissue transglutaminase in the developing chicken limb is associated both with apoptosis and endochondral ossification. Cell Death Differ 1999; 6: 146-154.

7. Jones RA, Nicholas B, Mian S, Davies PJ, Griffin M. Reduced expression of tissue transglutaminase in a human endothelial cell line leads to changes in cell spreading, cell adhesion and reduced polymerisation of fibronectin. $J$ Cell Sci 1997; 110 (Part 19): 2461-2472.

8. Barsigian C, Stern AM, Martinez J. Tissue (type II) transglutaminase covalently incorporates itself, fibrinogen, or fibronectin into high molecular weight complexes on the extracellular surface of isolated hepatocytes. Use of 2-[(2-oxopropyl)thio] imidazolium derivatives as cellular transglutaminase inactivators. J Biol Chem 1991; 266: 22501-22509.
TGF- $\beta$ superfamily and is a potent bone cell-differentiating factor as well as bone-formation stimulator. Our results showed that there is some relationship between BMP-2 and TG2. The stimulatory effect of TG2 regarding the osteoblast differentiation and mineralization may be possibly mediated in part by the up-regulation of BMP-2.

The present study established a specific linkage between TG2 and the differentiation and mineralization of human osteoblasts. TG2 levels and activity steadily increase and correlate with the expression of osteoblast differentiation markers during osteoblast maturation. When TG2 is inhibited, the program of SAOS-2 differentiation and mineralization is obstructed, implying that TG2 is essential for osteoblast differentiation. Moreover, the modulating effects of TG2 on osteoblasts could be related to BMP-2. Our finding is highly relevant to the understanding of the role of TG2 in the differentiation and mineralization of osteoblasts.

\section{Acknowledgments}

Research supported by the National Natural Science Foundation of China (\#81101334).
9. Aeschlimann D, Paulsson M. Transglutaminases: protein cross-linking enzymes in tissues and body fluids. Thromb Haemost 1994; 71: 402-415.

10. Johnson KA, Terkeltaub RA. External GTP-bound transglutaminase 2 is a molecular switch for chondrocyte hypertrophic differentiation and calcification. J Biol Chem 2005; 280: 15004-15012.

11. Orlandi A, Oliva F, Taurisano G, Candi E, Di Lascio A, Melino $\mathrm{G}$, et al. Transglutaminase-2 differently regulates cartilage destruction and osteophyte formation in a surgical model of osteoarthritis. Amino Acids 2009; 36: 755-763.

12. Johnson KA, van Etten D, Nanda N, Graham RM, Terkeltaub RA. Distinct transglutaminase 2-independent and transglutaminase 2-dependent pathways mediate articular chondrocyte hypertrophy. J Biol Chem 2003; 278: 18824-18832.

13. Nurminskaya M, Magee $C$, Faverman L, Linsenmayer TF. Chondrocyte-derived transglutaminase promotes maturation of preosteoblasts in periosteal bone. Dev Biol 2003; 263: 139-152.

14. Heath DJ, Downes S, Verderio E, Griffin M. Characterization of tissue transglutaminase in human osteoblast-like cells. $J$ Bone Miner Res 2001; 16: 1477-1485.

15. Wozniak M, Fausto A, Carron CP, Meyer DM, Hruska $\mathrm{KA}$. Mechanically strained cells of the osteoblast lineage organize their extracellular matrix through unique sites of alphavbeta3-integrin expression. J Bone Miner Res 2000; 15: $1731-1745$.

16. Al-Jallad HF, Nakano Y, Chen JL, McMillan E, Lefebvre C, Kaartinen MT. Transglutaminase activity regulates osteoblast differentiation and matrix mineralization in MC3T3-E1 osteoblast cultures. Matrix Biol 2006; 25: 135-148.

17. Bowness JM, Venditti M, Tarr AH, Taylor JR. Increase in 
epsilon(gamma-glutamyl)lysine crosslinks in atherosclerotic aortas. Atherosclerosis 1994; 111: 247-253.

18. Johnson K, Hashimoto S, Lotz M, Pritzker K, Terkeltaub R. Interleukin-1 induces pro-mineralizing activity of cartilage tissue transglutaminase and factor XIIla. Am J Pathol 2001; 159: 149-163.

19. Yin X, Chen Z, Guo Z, Liu X, Yu H. Tissue transglutaminase expression and activity in human ligamentum flavum cells derived from thoracic ossification of ligamentum flavum. Spine 2010; 35: E1018-E1024.

20. Rodan SB, Imai Y, Thiede MA, Wesolowski G, Thompson D, Bar-Shavit Z, et al. Characterization of a human osteosarcoma cell line (Saos-2) with osteoblastic properties. Cancer Res 1987; 47: 4961-4966.

21. Jeitner TM, Delikatny EJ, Ahlqvist J, Capper H, Cooper AJ. Mechanism for the inhibition of transglutaminase 2 by cystamine. Biochem Pharmacol 2005; 69: 961-970.

22. Xiaoxue $Y$, Zhongqiang C, Zhaoqing G, Gengting D, Qingjun
M, Shenwu W. Immortalization of human osteoblasts by transferring human telomerase reverse transcriptase gene. Biochem Biophys Res Commun 2004; 315: 643-651.

23. Cordell PA, Kile BT, Standeven KF, Josefsson EC, Pease RJ, Grant PJ. Association of coagulation factor XIII-A with Golgi proteins within monocyte-macrophages: implications for subcellular trafficking and secretion. Blood 2010; 115: 2674-2681.

24. Al-Jallad HF, Myneni VD, Piercy-Kotb SA, Chabot N, Mulani A, Keillor JW, et al. Plasma membrane factor XIIIA transglutaminase activity regulates osteoblast matrix secretion and deposition by affecting microtubule dynamics. PLoS One 2011; 6: e15893.

25. Nunes I, Gleizes PE, Metz CN, Rifkin DB. Latent transforming growth factor-beta binding protein domains involved in activation and transglutaminase-dependent cross-linking of latent transforming growth factor-beta. J Cell Biol 1997; 136: $1151-1163$ 